\title{
Mechatronic Experiments Course Design: A Myoelectric Controlled Partial-Hand Prosthesis Project
}

\author{
Ton-Tai Pan, Ping-Lin Fan, Huihua Kenny Chiang, Member, IEEE, Rong-Seng Chang, and \\ Joe-Air Jiang, Member, IEEE
}

\begin{abstract}
This paper describes a proposed laboratory project involving the design of a myoelectric-controlled partial-hand prosthesis to reinforce mechatronic education. The proposal focuses mainly on extract electromyogram (EMG) signals generated during contraction of the biceps. The EMG signals are first amplified and filtered by a laboratory-designed electronic circuit and then reprocessed using a microcontroller to drive the servomotor so that the designed prosthesis can be properly controlled. The project introduces students to component-level and system-level design and exposes them to the integration of a microcontroller, electronic circuits, sensors, and prosthesis mechanisms. Moreover, since the project results in a working prosthesis, student enthusiasm for mechatronic education increases, and they see its relevance to the field in applied engineering. Implementation of the laboratory project within the curriculum has been demonstrated to be highly motivational and educational and has even helped to attract more students to study mechatronic applications.
\end{abstract}

Index Terms-Electromyogram (EMG), laboratory curriculum, mechatronic education, microcontroller.

\section{INTRODUCTION}

A LL undergraduate students who major in Electrical Engineering (EE) and Bio-Industrial Mechatronics Engineering (BIME) at Kuang-Wu Institute of Technology (KWIT) and National Taiwan University (NTU), respectively, both in Taipei, Taiwan, are required to take one mechatronics course. The course syllabus originally focused on programmable logic controller-based control topics. Recently, the single-chip microcontroller has been one of the most widely used alternatives in industrial and consumer applications [1], [2]. Growing demand and the need for novel teaching materials has encouraged

Manuscript received March 14, 2002; revised July 8, 2003. This work was supported in part by the National Science Council of the Republic of China under Contract NSC 91-2133-E-002-113.

T.-T. Pan is with the Department of Electrical Engineering, Kuang-Wu Institute of Technology, Taipei 112, Taiwan, R.O.C. (e-mail: plfan@tea.ntptc.edu.tw).

P.-L. Fan is with the Graduate School of Toy and Game Design, National Taipei Teachers College, Taipei 106, Taiwan, R.O.C. (e-mail: plfan@tea.ntptc.edu.tw).

H. K. Chiang is with the Institute of Biomedical Engineering, National Yang-Ming University, Taipei 112, Taiwan, R.O.C. (e-mail: hkchiang@bme.ym.edu.tw).

R.-S. Chang is with the Institute of Optical Science, National Central University, Taoyuan 320, Taiwan, R.O.C. (e-mail: rschang@ios.ncu.edu.tw).

J.-A. Jiang is with the Department of Bio-Industrial Mechatronics Engineering, National Taiwan University, Taipei 106, Taiwan, R.O.C. (e-mail: jajiang@ccms.ntu.edu.tw).

Digital Object Identifier 10.1109/TE.2004.825528 the relevant faculties of the departments of KWIT and NTU to reorganize the contents of courses.

After some investigation, several topics in which the students were particularly interested were selected. These subjects included biomedical engineering applications, bio-robotics, and micro-stepping motor control. Accordingly, the design of a myoelectrically controlled partial-hand prosthesis has been included in the course content.

The disabled require special consideration during the design of special orthotic devices because of their particular needs. Early upper prosthesis limbs used simple spring resilience to control various knuckles, requiring the disabled to learn to control scapular joints to achieve the desired movement [3], [4].

Since conventional tools for those with disabilities have limited functions, as noted previously, artificial limbs are attractive to amputees. However, ingenious strategies for controlling the man-machine interface remain an interesting subject. The electromyogram (EMG) signal is the electrical physiological signal of activation of a motor unit associated with a contracting muscle and serves as a potential resource for a man-machine interface [5]-[7]. This project proposes a simplified controlling mechanism for teaching purposes. For simplicity, the system does not involve artificial intelligence techniques, such as neuro-fuzzy or genetic algorithms, which are left as advanced research topics for interested students at graduate school. The control mechanism is based on whether a muscle is contracting. Regardless of electrode allocation, hardware is necessary to detect waveforms and shape the measured EMG signals. The proposed partial-prosthesis mechanism is controlled by a built-in program. The palm will open when the EMG signals cease as the muscle is at rest. Although its only functions are opening and closing of the palm, the simplified prosthesis is still practical and is a favorable alternative to partial-finger function.

The proposed prosthesis consists of three main parts: 1) EMG signal-processing circuits; 2) the microcontroller and the embedded program; and 3) the prosthesis mechanism. This paper presents the information required to complete the described project and also details the system design. Moreover, this study suggests procedures for component selection, signal processing, prototype testing, and system integration. Retrospective assessments, results based on the instructor's observations, and interviews with students are also discussed. Finally, the primary findings from the delivery of the course and plans for improving the course are presented as conclusions. 
Signal Processing

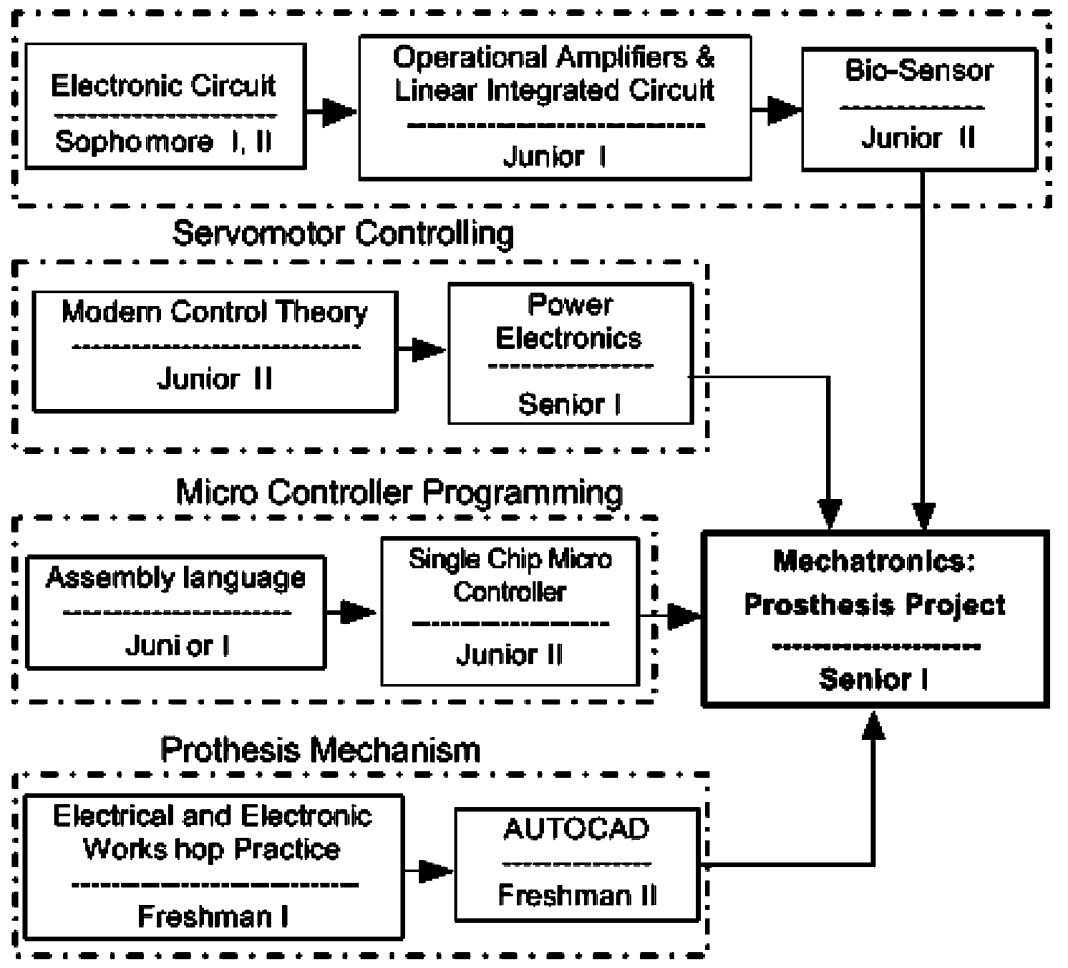

Fig. 1. Curriculum arrangement flowchart of prerequisites for the proposed mechatronics experiment project.

\section{DeVElopment PROCEDURE AND LABORATORY OBJECTIVES}

One important characteristic of this project is the high integration of subjects within the KWIT EE curriculum. This course, as part of the EE curriculum, provides hands-on experience, allowing students to use theoretical and practical knowledge learned between their freshman year and their senior year at KWIT's EE department. The primary prerequisites for the proposed mechatronics experimental project are knowledge of signal processing, servomotor control, microcontrollers, and prosthesis mechanism design. Fig. 1 presents the arrangement of the integrated courses in the curriculum, in flowchart form.

This project is designed as part of the laboratory course in mechatronics. Meanwhile, the prosthesis is designed to extract EMG signals from wrist disarticulation patients to replace some finger function. The detected EMG signals must first be processed, digitized, and converted to pulsewidth modulation (PWM) signals, which are then used to control the designed prosthesis mechanism. The knowledge required to implement the proposed prosthesis design project thus covers signal-processing techniques, microprocessor interface design, and a scheme for controlling a servomotor mechanism. Many educational issues are addressed, including signal acquisition and processing, circuit design, component selection, microprocessor interface design, prosthesis mechanism design, assembly language programming, and control scheme development. The goals of this laboratory course are as follows:

1) reinforce the operational amplifier (op amp) circuit design techniques taught in electronic circuit courses;

2) strengthen microcontroller programming skills and interface circuit design abilities;
3) familiarize students with computer-aided design or computer-aided manufacture software packages, such as $\mathrm{Au}-$ toCAD;

4) teach students about remote control (R/C) servomotor control;

5) provide students with the workbench techniques and background in translation mechanisms necessary to pursue more sophisticated design projects.

To improve the content of the course and make it more appealing to students, the content will be modified in the near future to include additional topics such as the digital control mechanism, fuzzy logic control, and PWM switching power circuits.

\section{Design Methodology}

The pedagogy for this course has been discussed in detail by the curriculum committee of the EE department at KWIT. Although the pedagogical method will vary in complexity, the basic principles to be followed remain the same. To promote problem solving, pedagogical processes are arranged as follows: defining the problem; finding possible solutions; evaluating and correcting the solutions; optimization; and implementation. Thus, before running the course project, the instructor should provide the students with an overview of mechatronic design methodology. Shetty and Kolk clearly described these topics in their textbook [2]. Based on the pedagogical processes and following the methodology in [2], the authors considered the mechatronic design process to include three phases-modeling, prototyping/testing, and deployment. These three phases are described subsequently. 


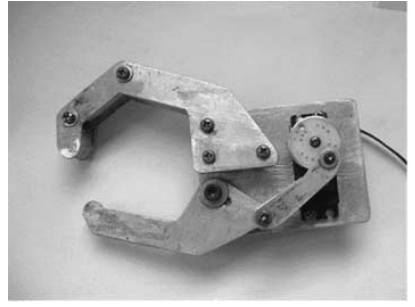

(a)

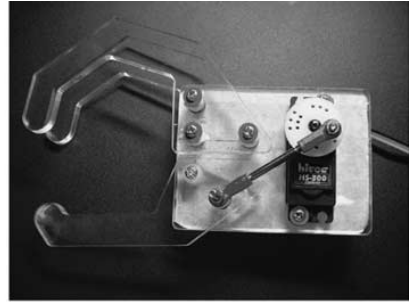

(b)
Fig. 2. Photographs of the proposed prosthesis showing (a) an aluminum type and (b) an acrylics type.

Modeling. The first step is to analyze the goals of the project and the technical environment in which the system is integrated. Normally, a block diagram is used to create intuitively understandable behavioral models of the system. In this case, the prosthesis system can be considered to combine bio-signal acquisition and processing, controlling, and translating subsystems. Each subsystem can be modeled separately.

Prototyping/testing. Actual hardware is used to replace part of the model of each subsystem. On-board diagnoses of the signal processing, controlling, and translating subsystem should be made in this phase. Each subsystem can be built and tested individually.

Deployment. The control code used on the embedded processor of the final product is coded, and the subsystems are connected to complete the full system design.

These three phases can be repeated until the results are satisfactory. In addition, the difference between a mechatronic system that involves concurrent engineering and a traditional multidisciplinary system design that employs a sequential design-by-discipline approach should be emphasized.

\section{SYSTEM IMPLEMENTATION AND RELATED LEARNING ACTIVITIES}

\section{A. System Descriptions}

Fig. 2 presents photographs of the proposed prosthesis. Fig. 3 shows a block diagram of the myoelectrically controlled partial-hand prosthesis system. The electrodes are attached to the biceps. Biceps consist of bundles of skeletal fibers.

When the fibers extend along the length of the muscle, the extracellular field potential is evoked [7]. The extracellular field potential is an EMG and has a brief duration of 3-15 ms. The typical amplitude of EMG ranges from $20-2000 \mu \mathrm{V}$, depending on the size of the motor unit and the position of the electrode. The EMG signals generated from a contracting muscle and detected by physiological signal electrodes are first sent to the instrumentation amplifier, the bandpass filter, and the precision rectifier circuits. Following amplification, filtering, and rectification, the resulting signals are used as inputs to the microcontroller and are converted to digital ones by a 1-b analog comparator embedded in the microcontroller. According to the digital signals, the program built in the microcontroller can make precise decisions and then output PWM signals to control the $\mathrm{R} / \mathrm{C}$ servomotor to drive the prosthesis. The functions associated with each part of this system are detailed subsequently.

\section{B. EMG Signal-Processing Circuits}

Fig. 4 shows the EMG signal-processing circuit. Three electrodes are required to acquire the EMG signals. Two of these electrodes (electrodes I and II, or EMG I and II in the figure) are attached to the biceps and serve as the differential inputs to the instrumentation amplifier, while the third (ground, GND) is arbitrarily attached to a different location on the arm as a ground reference and is connected to the ground of the system. The EMG indicator light-emitting diode (LED) lights up when EMG signals are detected.

Electrodes can be categorized as either passive or active. After several iterations of pretesting electrode efficiency, passive electrodes were adopted in this project for economy and convenience. The selected electrodes must meet the following requirements:

1) include conductive adhesive hydrogel;

2) have a high-quality foam substrate that resists fluids and conforms easily to the skin, to ensure excellent trace quality;

3) be small for convenient lead placement;

4) be teardrop-shaped for easy release and removal;

5) have a perforated liner that allows the electrodes to be divided into strips;

6) support multiple packaging configurations.

At this stage, the instructor should explain the difference between the two types of electrodes and explain why the passive one is preferred. The students' design efforts should focus on EMG signal acquisition and op amp postprocessing circuits.

After attaching the electrodes, the students first make observations and records of the EMG signals from the oscilloscope output by the circuits on the breadboard. Since the amplitudes of the raw EMG signals thus obtained are too small for further processing by the microcontroller, these signals must be processed before they are fed into the microcontroller. Before these signals are processed, the instructor should explain the typical characteristics of the physiological signals to the students, as shown in Fig. 5.

From Fig. 5, except for amplifying raw EMG signals to reject common-mode signals using differential amplifiers, a bandpass filter is required to increase the signal-to-noise ratio and reject other physiological signals, such as the electrocardiogram (ECG) signal and axon action potential (AAP). Fig. 4 presents the circuit diagram for amplifying and filtering the signals.

The Burr-Brown INA-118P [8] amplifier is used as a firststage differential amplifier with a gain of 20. This amplifier exhibits a high common-mode rejection ratio (CMRR) and effectively reduces noise [9], [10]. This component is also selected for its compactness. Differential inputs allow the direct current (dc) component to be eliminated from the electrodes. A bandpass filter with gain $=150$, consisting of a high-pass and a low-pass filter, was designed with a low power op amp LF351 (National Semiconductor) [11]. The cutoff frequency of the low-pass filter was $500 \mathrm{~Hz}$ while that of the high-pass filter was $50 \mathrm{~Hz}$. Meanwhile, the total gain of the combination of the instrument amplifier and the bandpass filter was $20 \times 150=3000$. This gain is high enough to amplify the obtained EMG signals to a level suitable for processing during 


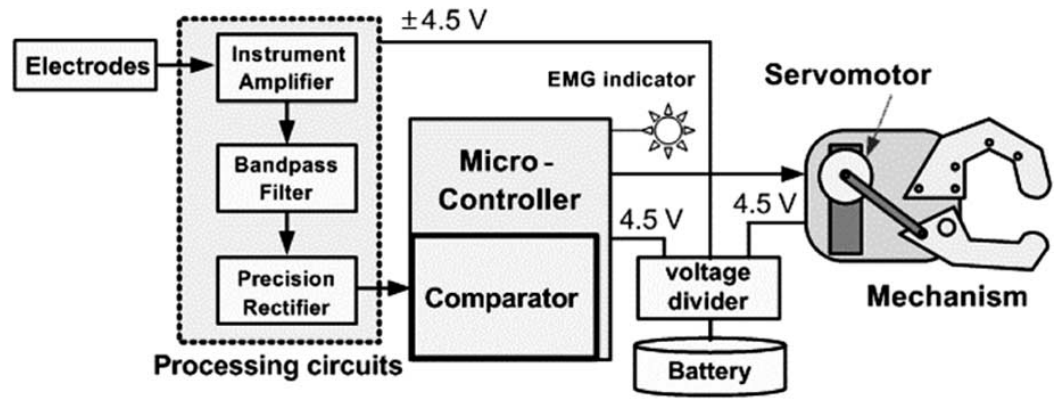

Fig. 3. Block diagram of the proposed prosthesis system.

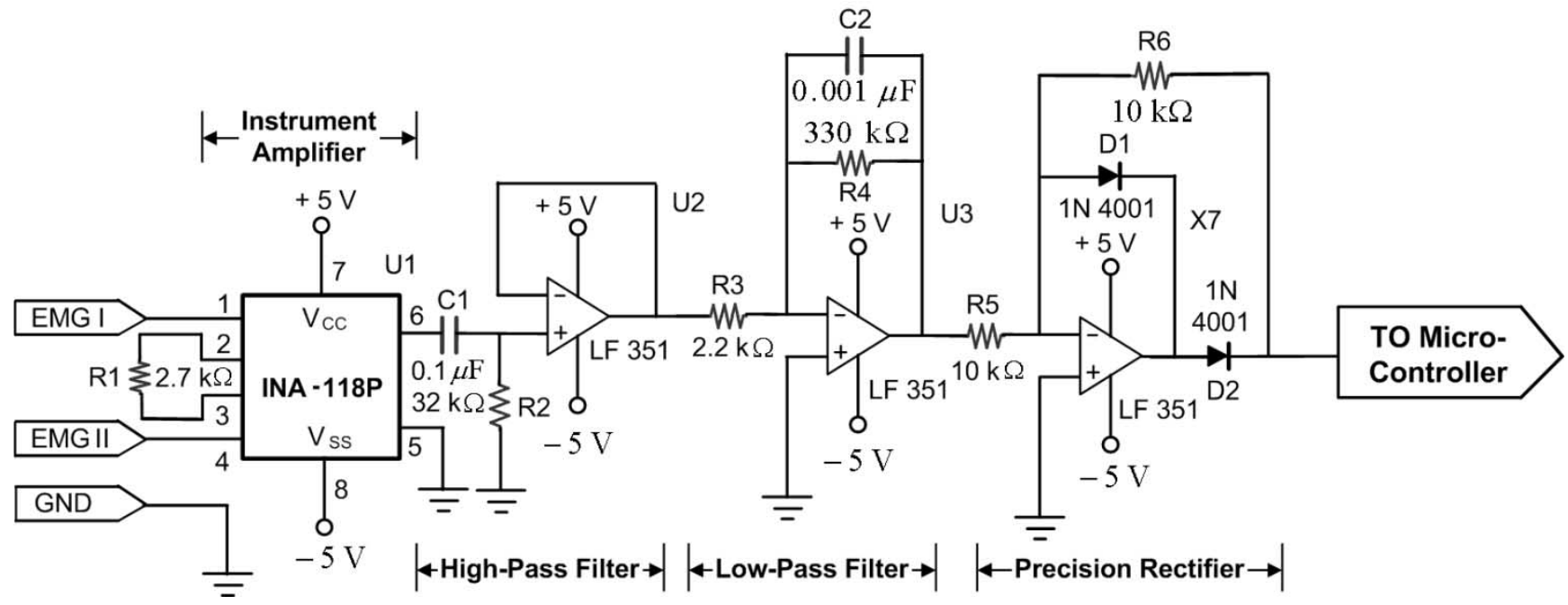

Fig. 4. Measurement and processing circuit for EMG signals.

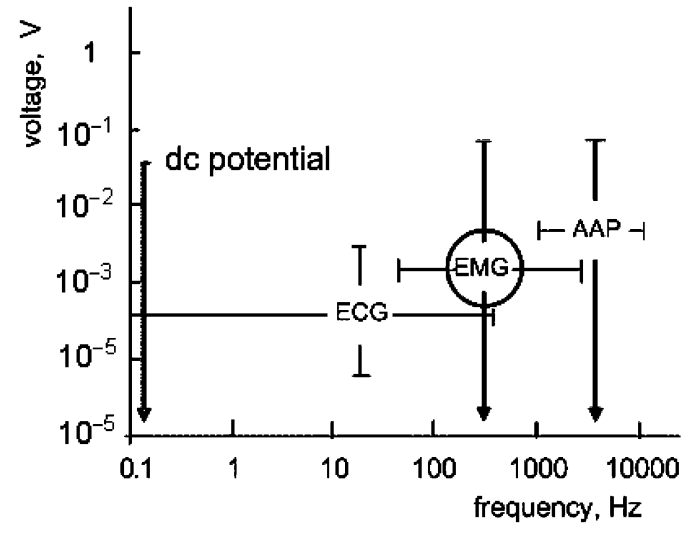

Fig. 5. Typical characteristics of the EMG signal.

the subsequent precision rectifier stage. After the signal passes through the bandpass filter, the precision rectifier reshapes the pulses that can be fed successively into the comparator embedded in the microcontroller. During this phase, the instructor asks students to record EMG output signals for different electrode locations and various strengths of muscle.

Fig. 6 presents the typical waveforms measured at the last stage of the signal-processing circuit. Each spike in the figure represents a muscle-firing event. Since the muscle strengths are different between male and female, the EMG signals between male and female students are also observed.

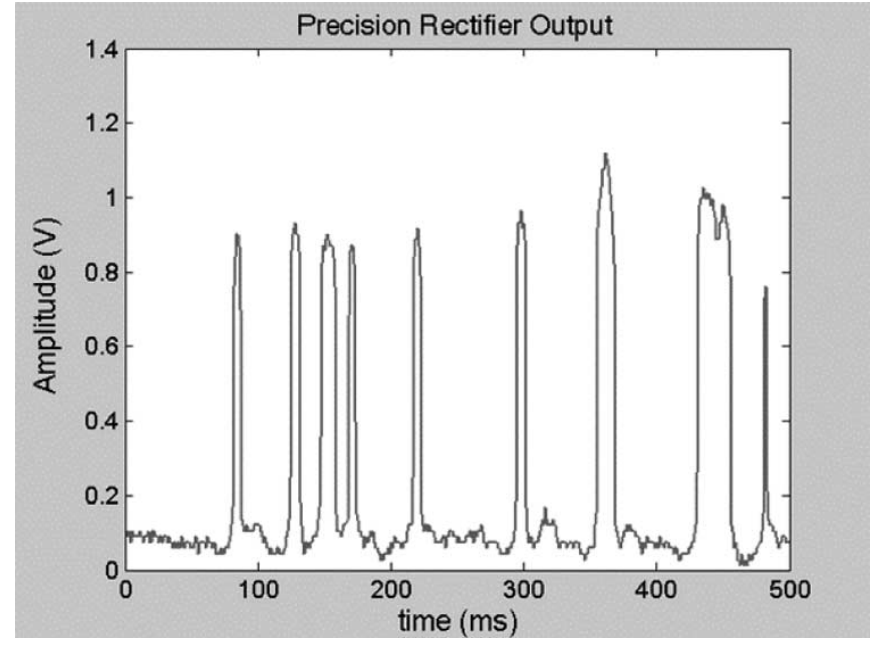

Fig. 6. EMG waveforms measured at the output terminal of the precision rectifier.

Since the students have taken a coordinated series of electrical circuitry and electronics courses at sophomore and junior level, they have a clear understanding of the aforementioned circuits and can complete the processing circuits without problems. However, the instructor should emphasize the criteria by which the components are selected to meet the requirements of small current consumption-compactness, the suitability of the bandwidth of the bandpass filter given the characteristics of the EMG 


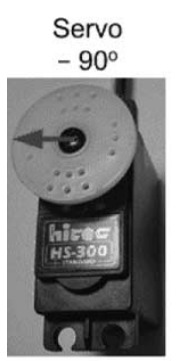

$0.5 \mathrm{~ms}$

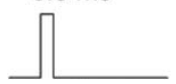

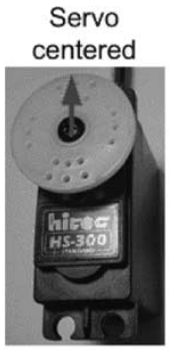

$1.5 \mathrm{~ms}$

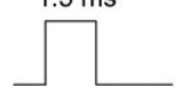

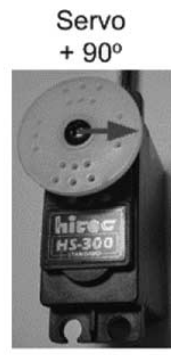

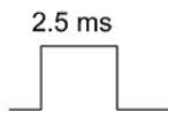

Fig. 7. Servomotor shaft position and corresponding required pulsewidths.

signals, and the gain factor design considerations of each stage of the circuit.

To save energy and for convenience, a single $9-\mathrm{V}$ battery is used to power the entire system. Moreover, a resistor divider is utilized to generate +4.5 and $-4.5 \mathrm{~V} \mathrm{dc}$; both can serve as dc sources for op amps in a signal-processing circuit. The $+4.5-\mathrm{V}$ source also supplies the microprocessor and the servomotor. Notably, complementary metal-oxide-semiconductor (CMOS)-based components, such as INA-118P, LF351, and the Atmel AT89C1051 8-b microcontroller [12], are used throughout the design to ensure that the designed prosthesis mechanism can be driven by a low-voltage and low-current servomotor.

\section{Remote Control Servomotor and Microcontroller Programming}

The power consumption and compactness must be considered so that selecting a motor is challenging. A servomotor commonly used for control applications consumes much current and is oversized and expensive. Because a stepping motor loses step under some conditions, it unsuitable in this application. Besides, servomotors and stepping motors must be controlled by external driver circuits. In particular, the motors and their drivers are too expensive for a laboratory that includes 25 stations. Following a survey of products, an R/C servomotor (model HS-300 by Hitec, Poway, CA) [13], usually used for the R/C of a boat or airplane, is adopted herein to drive the prosthesis mechanism. The R/C servomotor, equipped with a position feedback control circuit and a decelerating gearbox assembly, provides a simple control mechanism. (The internal construction of the decelerating gearbox assembly and the feedback potentiometer of servomotor are given in [13].) The R/C servomotor is controlled by a PWM signal, which can drive the motor to a desired position according to the width of the pulse. In this design, the Mitsubishi M51660L control chip is adopted as an R/C servomotor controller. (For the details of the driver circuit, refer to [14].) Fig. 7 shows the shaft positions of the servomotor and the corresponding required pulsewidths.

Given a $0.5-2.5-\mathrm{ms}$ pulsewidth, the R/C servomotor can rotate from $-90^{\circ}$ to $+90^{\circ}$ clockwise. The output shaft of the servomotor can drive the linkage so that the movable part of the prosthesis rotates with respect to the swivel and then closes the palm of the prosthesis. The output torque of the servomotor is approximately $3 \mathrm{~kg}-\mathrm{cm}$ so that the designed prosthesis can

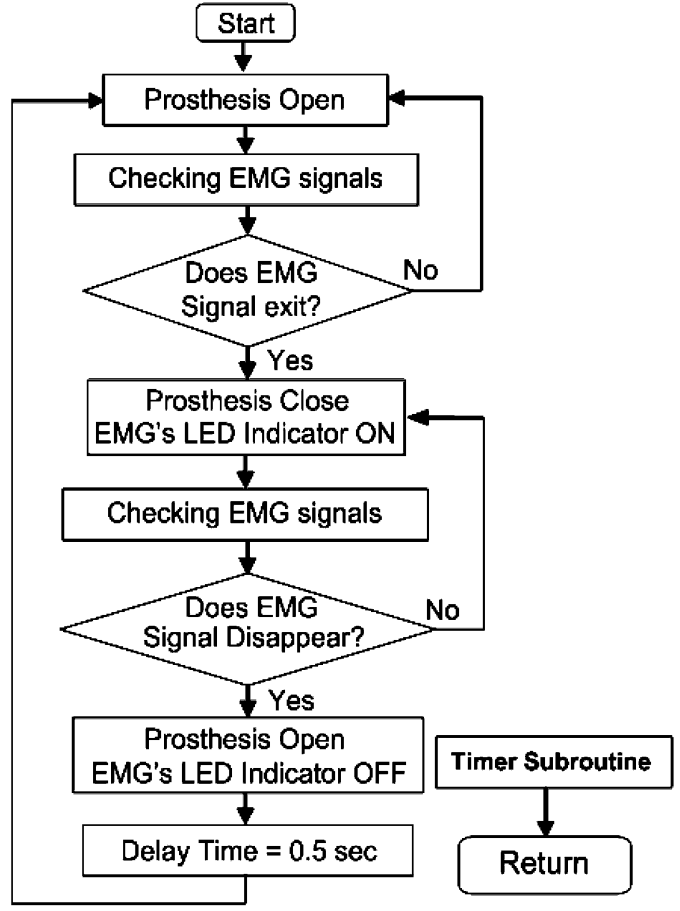

Fig. 8. Programming flowchart of microcontroller for the R/C servomotor.

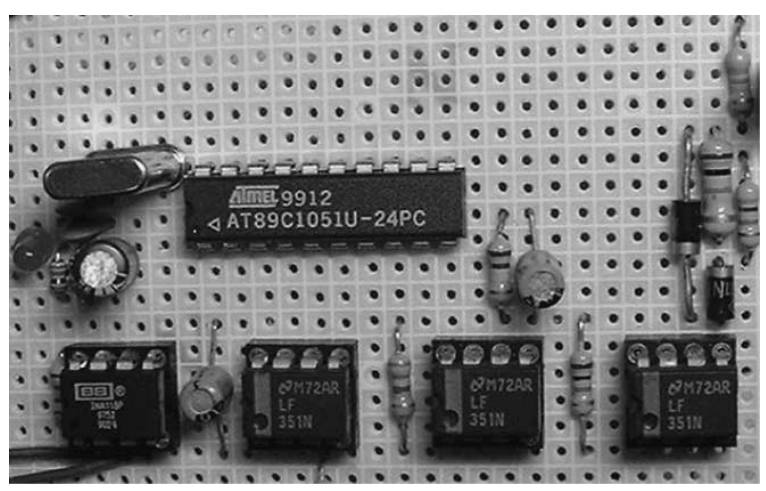

Fig. 9. Photograph of the circuit board for the microcontroller of the proposed prosthesis.

easily grasp an object that weighs $1 \mathrm{~kg}$. The students can use a microcontroller to easily generate an appropriate pulsewidth. A CMOS-based 8-b Atmel AT89C1051 single-chip serves as the microcontroller of the system; it accepts the processed EMG signals and is programmed to control the $\mathrm{R} / \mathrm{C}$ servomotor. The 1-b embedded analog comparator allows the processed analog EMG signals to be converted into digital ones. The conversion rate of the 1-b comparator is $10 \mu \mathrm{s}$. Fig. 8 shows a flowchart of the software design.

Except the EMG signal-checking loop, opening and closing the prosthesis are the basic movements of the system. The instructor and the students begin by discussing how to generate a pulse of an appropriate width to control the servomotor when EMG signals are detected. Two approaches are proposed. One is to use a time-delay subroutine in the EMG signal-checking loop. This approach is straightforward but wastes the computing resources of the microcontroller. Another approach is to use an interrupt-service routine. When EMG signals appear at the external interrupt pin of the microcontroller, an interrupt-service 


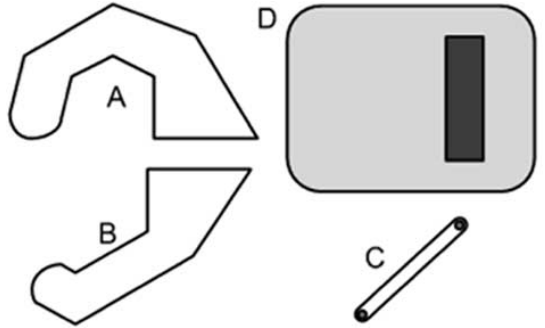

(a)

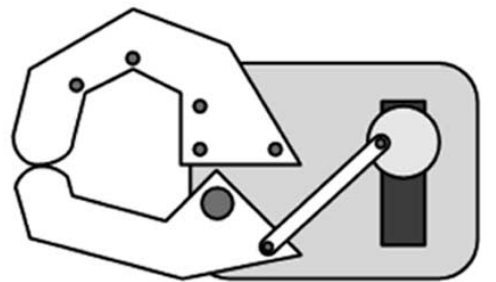

(b)

Fig. 10. (a) Explosion and (b) assembly of the designed prosthesis.

routine is activated to generate suitable pulses. Although students can complete the necessary tasks by either of these two approaches, they typically consider the interrupt service routine approach to be better than the loop instruction approach for performing a complicated control task.

Simulation software, such as Sim51, or the in-circuit emulator (ICE), helps programming during the development and testing phases. An oscilloscope is provided to monitor the pulsewidths generated by the microcontroller. Students can modify the program, after observing the signals on an oscilloscope, to yield the desired pulsewidths.

After these two parts are completed, the students can integrate the circuits into a single-board system by soldering. Fig. 9 depicts the resulting circuit board.

\section{Mechanism}

Making the mechanism of the prosthesis is a rather difficult part of the experiment since most students who major in EE are not competent machinists. To reduce the impact of this weakness, the components of the prosthesis are all made of aluminum or acrylics, since these materials are easy to reprocess and allow components to be made on a simple workbench. Fig. 10 shows the AutoCAD files, named explosion.bmp and assembly.bmp, which are the exploded and assembly drawings, respectively, of the components of this EMG prosthesis. The components of the prosthesis mechanism include a fixed part (A), a movable part (B), a linkage (C), and a main body (D). Using the actual-size data provided by the instructor, students can easily make all the parts of the prosthesis using hand tools. Furthermore, the students can draw the components using AutoCAD and convert the drawing to a DXF file for processing by a computer numerical control (CNC) milling machine. Since the KWIT Mechanical Engineering Department has CNC milling machines, students are encouraged to learn to operate the cutting machine to complete their tasks.

The final part of the experiment is the integration of the system and the testing of the completed prosthesis. Students try to control the prosthesis by contracting their biceps to make it grasp an object, such as the vessel, as shown in Fig. 11, to measure its effectiveness.

\section{OBSERVATIONS AND RESPONSES}

This laboratory project requires approximately four to five 3-h laboratory sessions. Almost 200 students have participated

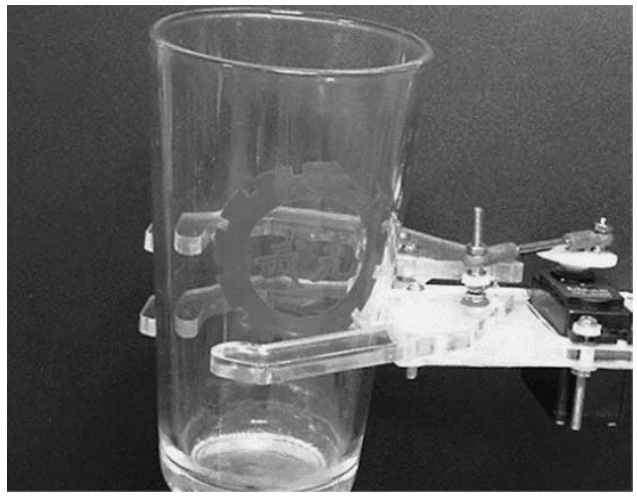

Fig. 11. Illustration for the designed prosthesis mechanism to grasp and lift up a vessel weighing about $120 \mathrm{~g}$.

in this project over the past two years and have offered very positive comments.

In this course, each student must implement his/her own prosthesis system. The lecturer constantly interacts with the students and helps them to debug their systems. Students are free to ask questions in the classroom, in the laboratory, or via e-mail, allowing the lecturer to respond to the questions immediately. Most students successfully complete their projects in time. Following frequent interactions with the students, the instructor records their comments.

Students are individually surveyed to evaluate the design methodology. Students were asked to rate five qualities on a four-point scale. Assessment based on the instructor's observations and interviews with students increase the objectivity of the overall assessment.

Table I shows the results of evaluation for the class of 2002 at KWIT's EE department (obtained four months after taking the course). Eighty-nine students responded.

As shown in Table I, more than $90 \%$ of the students considered this project to be interesting. According to the interviews, this course, with such original teaching materials, was the most enlightening one they took during their studies. Students felt that processing bio-signals was a new experience, which considerably promoted their understanding of bio-systems. After the students had completed the laboratory project, the instructor observed that the students were enthusiastic about the practical operation of their projects, and they tried to grasp various nearby objects using their prosthesis systems. Students seemed to be satisfied with their own prosthesis projects.

Table I also shows that $88 \%$ of the responding students furthered their understanding of prerequisites. Many interviewed 
TABLE I

Student Responses on the Prosthesis Project

\begin{tabular}{l|c|c|c|c}
\hline \multicolumn{1}{c|}{ Questions } & I & II & III & IV \\
\hline I found this project interesting. & $6 \%$ & $86 \%$ & $4 \%$ & $4 \%$ \\
\hline This project increased my understanding of prerequisites. & $10 \%$ & $78 \%$ & $7 \%$ & $5 \%$ \\
\hline This project gave me experience of system-level design. & $15 \%$ & $75 \%$ & $6 \%$ & $4 \%$ \\
\hline This project improved my integration capability. & $14 \%$ & $76 \%$ & $5 \%$ & $5 \%$ \\
\hline This project gave me useful mechanical workshop skills. & $20 \%$ & $60 \%$ & $13 \%$ & $7 \%$ \\
\hline I: I strongly agree. II: I agree. III: I disagree. IV: I strongly disagree. \\
\hline
\end{tabular}

students stated that such a linkage to the prerequisites surely contributed to their overall academic growth and allowed them, practically, to apply learned knowledge in future work. The instructor felt that the interviewed students gained confidence in their abilities to apply their learned knowledge. Some students also stated that the project taught them how to design a system by modeling it into smaller subsystems through the block diagram method. The students stated that they could easily complete the analysis and design tasks using the block diagrams.

The survey revealed that $91 \%$ of the students were confident in their ability to integrate and perform system-level design after they completed their project. Some interviewed students told the instructor that the project provided them with hands-on experience of incorporating signal processing, controlling a servomotor, programming a microcontroller, and using a translation mechanism. This multidisciplinary experience contributed to their understanding of integration concepts for designing a system, in contrast with traditional experimental courses, which typically focus on a single area. Students mentioned that prototyping/testing each subsystem was important and useful in designing an integrated system. In particular, they responded that they gained knowledge of debugging techniques in this design methodology. The project also taught them how hardware and software function together. Such training really helped them to deploy and implement a system like the one in this project. These responses were consistent with the observations of the supervisors of the final-year design project. The supervisors noted the students' willingness to integrate hardware/software into their projects, rather than just construct hardware or software on its own.

The survey also found that $80 \%$ of students learned useful mechanical workshop skills. The interviewed students mentioned that training in the operation of simple workbench or mechanical tools broadened their view of engineering. The instructor also noted that students were satisfied with the components of the prosthesis mechanism that they themselves implemented. However, some students said that they did not need such mechanical training in their study of EE.

This laboratory project has been run for two consecutive years at KWIT. The introduction of this prosthesis experiment has increased students' understanding of mechatronics and stimulated their interest in the area of mechatronic applications. The experiment smoothly integrates the topics of electronic circuit design, microcontroller programming, sensing, and translation mechanisms, all of which are included in the EE curriculum.
This project has become a popular one, and some senior students are working on theses that utilize skills acquired on this course. Some of these thesis projects are as follows:

1) differentiating the EMG signals into more states;

2) improving the output torque of the designed prosthesis;

3) studying EMG signals from various locations of muscles.

The instructors observed considerable enthusiasm among the students. Most of the students appeared highly motivated to tackle difficult experimental problems during the course and became increasingly aware of system integration design skills. Moreover, students gained increased confidence in their abilities to utilize the knowledge obtained from other courses they had taken. Consequently, this laboratory project helps to make the educational experience more interesting and prepares students for solving practical system integration problems, which they may encounter in field applications.

\section{CONCLUSION}

This paper presents a project to develop a myoelectrically controlled, partial-hand prosthesis. This project is Part I of a laboratory course developed by the KWIT EE and the NTU BIME Curriculum Study Committees. The project is designed for inclusion in a one- to two-semester mechatronics course at undergraduate level, and its central theme is the idea that mechatronics is part of the basic fabric of modern technology. Given this theme, the authors endeavored to demonstrate how the analysis and design of mechatronics is inseparable from the ability to design complex electronics, microprocessors, machinery, control systems, and consumer products. Specific aims distinguish this project from conventional ones; they include building an explicit understanding of concepts and ideas in terms of previous learnings, emphasizing the relationship between conceptual understanding and problem-solving approaches, giving students practice in using various software packages, and providing them with a solid foundation in foretaste of practical mechatronics. Furthermore, the proposed laboratory project introduces students to biosensors, myodynamics, and biomedical engineering. The topics covered by the proposed project correspond to the coverage of the modern mechatronics curriculum. Striving for clarity and consistency above all else, the designed project has been demonstrated to be highly motivational and educational. Survey results indicate that most students (more than $80 \%$ ) respond that this project not only furthers their understanding of prerequisites, but also equips them with useful mechanical 
workshop skills. They gain confidence in their abilities to integrate and perform system-level design.

The prosthesis project is at its midpoint approximately. While the survey results seem to indicate that the project has successfully met its objectives as the authors expected, the project remains under continuous development. The authors are planning a weightlifting prosthesis competition at KWIT's EE department over the coming years. The authors' experience of other courses suggests that such a design contest will provide students with useful design experience and motivate the development of new courseware materials for the faculty. The prostheses project course will be improved by large-scale student participation in such a competition.

\section{REFERENCES}

[1] C. Fraser and J. Milne, Integrated Electrical and Electronic Engineering for Mechanical Engineers. New York: McGraw-Hill, 1994.

[2] D. Shetty and R. A. Kolk, Mechatronics System Design. Boston, MA: PWS-Kent, 1997.

[3] D. G. Shurr and T. M. Cook, Prosthetics and Orthotics, 2nd ed. Englewood Cliffs, NJ: Prentice-Hall, 2002, pp. 150-155.

[4] C. C. Nielsen, "A survey of amputees: Functional level and life satisfaction, information needs, and the prosthetist's role," J. Pros. Orth., vol. 3, pp. 125-129, 1989.

[5] W. Ganong, Review of Medical Physiology, 20th ed. New York: McGraw-Hill, 2001, pp. 72-74.

[6] J. H. Kuo, "Development of a controller for artificial arm via EMG pattern recognition," M.S. thesis (in Chinese), Institute of Electrical Engineering, National Taiwan Univ., Taipei, 1995.

[7] J. G. Webster, Medical Instrumentation: Application and Design, 3rd ed. New York: Wiley, 1998, pp. 121-182.

[8] Burr-Brown IC Data Book, Linear Products, pp. 4.124-4.134, 1997.

[9] A. S. Sedra and K. C. Smith, Microelectronic Circuits, 4th ed. New York: Oxford Univ. Press, 1998.

[10] S. Franco, Design With Operational Amplifiers and Analog Integrated Circuits. New York: McGraw-Hill, 1988.

[11] National Semiconductor, Linear Databook, vol. 1, pp. 2.38-2.45, 1987.

[12] ATMEL, Small Footprint Reprogrammable Flash Micro-Controller AT89C1051U Data Sheets, pp. 1-15, 2001.

[13] D. Sawicz, Hobby Servo Fundamentals. Poway, CA: Hitec, 2001, pp. $1-10$.

[14] Mitsubishi Semiconductor, M51660L RC Servo Controller Datasheet, Tokyo, Japan, pp. 3-4, 2001.
Ton-Tai Pan was born in Taipei, Taiwan, in 1960. He received the B.S. and M.S. degrees in automatic control engineering from Fung-Chia University, Taichung, Taiwan, R.O.C., in 1982 and 1985, respectively.

He worked as an Assistant Scientist in the Guidance and Control Group at Chung-Shan Institute of Science and Technology from 1985 to 1992. He is now an Assistant Professor in Electrical Engineering at Kuang-Wu Institute of Technology, Taipei, Taiwan, R.O.C. His current research interests include automatic control and medical ultrasound imaging.

Ping-Lin Fan was born in Taipei, Taiwan, on December 29, 1961. He received the B.S. degree in electrical engineering, the M.S. degree in optical science, and the Ph.D. degree in optical science from the National Central University, Chung-Li, Taiwan, R.O.C., in 1985, 1987, and 2002, respectively.

From 1989 to 2003, he was at the Kuan-Wu Institute of Technology, Taipei, Taiwan, R.O.C. He then came to the National Taipei Teachers College, Taipei, Taiwan, R.O.C., where he is now an Associate Professor of the Graduate School of Toy and Game Design. His current research interests include mechatronics and computer game design.

Huihua Kenny Chiang (S'91-M'92) received the B.S. degree in electrical engineering from the National Tsing-Hua University, Hsinchu, Taiwan, R.O.C., in 1982 and the M.S. and Ph.D. degrees in electrical engineering from the Georgia Institute of Technology (Georgia Tech), Atlanta, in 1987 and 1991, respectively.

In 1992, he was employed as a Research Scientist at the Georgia Tech Research Institute, GA. In 1993, he joined the Institute of Biomedical Engineering, National Yang-Ming University, Taipei, Taiwan, R.O.C., as an Associate Professor. In 1999, he became Professor of Biomedical Engineering at the same institute. His current research interests include medical ultrasound signal processing, noninvasive optical diagnostic techniques, and cardiac signal visualization and processing.

Rong-Seng Chang received the M.S. degree in lasers and electrooptics from the Hebrew University of Jerusalem, Jerusalem, Israel, in 1972 and the Ph.D. degree from the Optical Sciences Center, University of Arizona, Tucson, in 1982.

$\mathrm{He}$ is currently a Professor at the Institute of Optical Science, National Central University, Chung-Li, Taiwan, R.O.C. His current research interests are in optical design, image processing, and biochips.

Joe-Air Jiang (M'01) was born in Tainan, Taiwan, R.O.C., in 1963. He received the B.S. degree from the National Taipei University of Technology, Taipei, Taiwan, R.O.C., in 1983 and the M.S. and Ph.D. degrees in electrical engineering from the National Taiwan University, Taipei, R.O.C., in 1990 and 1999, respectively.

From 1990 to 2001, he was at the Kuang-Wu Institute of Technology, Taipei, Taiwan, R.O.C. He then came to National Taiwan University, Taipei, R.O.C., where he is now an Assistant Professor of bio-industrial mechatronics engineering. His area of interest is in computer relaying, mechatronics, and bioeffects of electromagnetic waves. 